\title{
EVALUATION OF CERVICAL SCREENING COVERAGE IN KALUTARA DISTRICT
}

\author{
D T P Liyanage ${ }^{1}, \mathrm{R}$ De A Seneviratne ${ }^{2}$
}

\section{Abstract \\ Objective:}

To determine the cervical screening coverage of women 40-60 years in Kalutara District since the inception of the programme in 1996.

\section{Methodology:}

Cervical screening which is conducted in Well Woman Clinics (WWC), involves both visual inspection of the cervix and Pap smear testing. A descriptive cross sectional survey was conducted to assess the cervical screening coverage achieved since the inception of the programme and to assess the quality of services. The area investigated was the Kalutara district and individual $\mathrm{MOH}$ (Medical Officer of Health) areas within it. Data of screened females between 33 to 63 years were obtained from the records maintained in the WWClinics

\section{Results}

The results were expressed as a percentage of the total 40-60 females in the District and individual MOH areas. The cervical screening coverage in Kalutara District was $2.2 \%$ since the inception of the programme. Although the coverage was low, a rising trend of cervical screening from 1996 was observed particularly after the year 1998. Only three $\mathrm{MOH}$ areas out of 7 conducted more than 4 WWClinics per month, which is the recommendation of Ministry of Health. Seventy four percent (1135) of total screenings (1535) were performed in those three clinics. Only one MOH area conducted an out-reach clinic. Five $\mathrm{MOH}$ areas conducted the clinics in combination with Maternal and Child Health/Family Planning (MCH/FP) clinics, while the other two $\mathrm{MOH}$ areas conducted separate WWClinics. The highest cumulative coverage was observed in Bulathsinhala $\mathrm{MOH}$ area $(9.3 \%)$ where there were more than 4 WWclinics per month and clinics were conducted separately.

\section{Conclusion}

The low cervical screening coverage of $2.2 \%$ over a 5 year period in Kalutara District resembled the cervical screening coverage pattern in other developing countries. Cervical screening coverage could be increased by increasing frequency and number of clinics especially in the periphery and conducting WWClinics separately without combining with $\mathrm{MCH} / \mathrm{FP}$ Clinics.

Key words

Cervical screening, Well Woman Clinics, evaluation, coverage

\section{Introduction}

Cervical cancer is a major health problem among females in Sri Lanka. It accounts for $17.7 \%$ of all cancers among females (1). It is the second commonest malignancy among females and the incidence was 6.6 per $10^{5}$ population in 1995 (2) and shows a rising trend (Figure I).

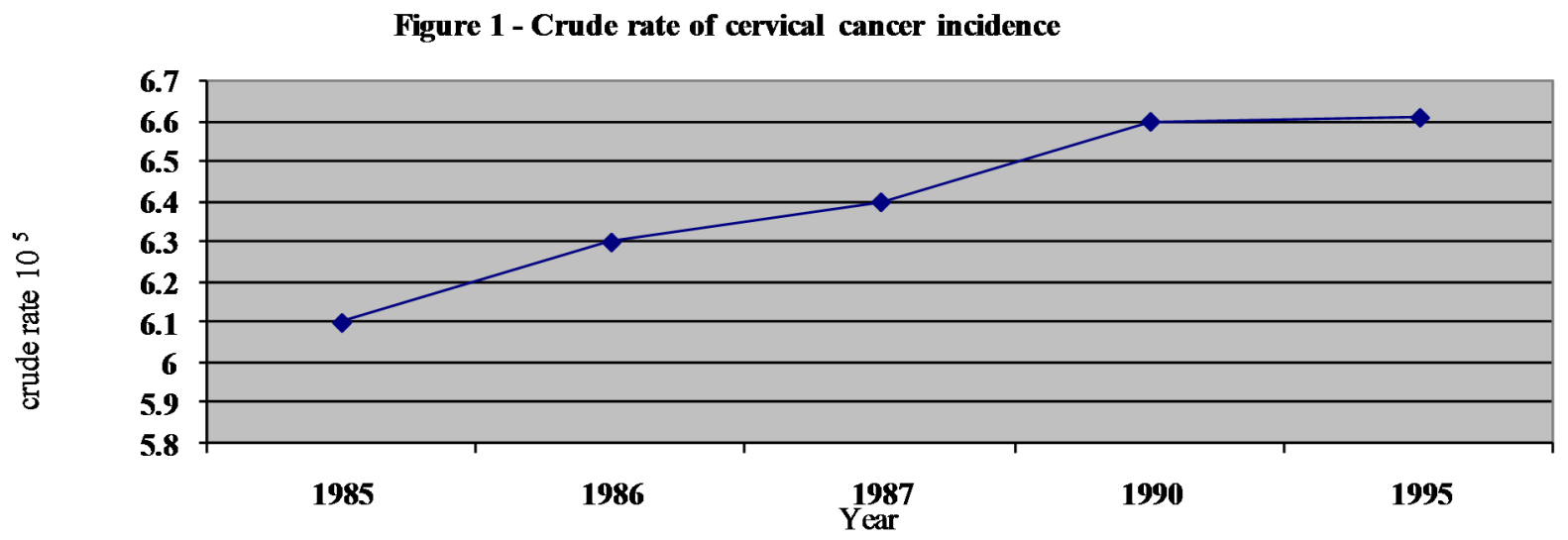

Crude rate (Number of cervical cancer cases per $10^{5}$ mid year population at risk)

Source: Cancer Registry (1990)

1. Consultant Community Physician, National Institute of Health Sciences, Kalutara

2. Professor in Community Medicine, Department of Community Medicine, Faculty of Medicine, Colombo

Correspondence : trooph123@yahoo.com 
Prevention of cervical cancer depends on early detection of cases through screening and treatment by radical surgery and radiotherapy. The 5-year survival rate is virtually $100 \%$ for carcinoma in situ while in cases diagnosed in an advanced invasive stage it is less than $35 \%$ (3).

Pre cancerous stages in carcinoma of cervix exist for long duration time. Hence, this disease is appropriate for detection by a screening program at very early stages. Early detection of precancerous stages by wellorganized screening programmes has been shown to be effective in reducing morbidity and mortality from the disease. In Nordic countries, Iceland, Finland and Sweden the cumulative cervical cancer mortality rates fell by $84 \%, 50 \%$ and $34 \%$ respectively between 1965 and 1982(4) following the screening programmes.

The Ministry of Health of Sri Lanka with the assistance of United Nations Population Fund (UNFPA) set up 300 WWClinics in the country to conduct cervical screening. The first WWClinic was established in June 1996 in the Kalutara District.

The objective of the cervical screening programme is to reduce the morbidity and mortality from the disease. Hence, at the completion of five years of the programme it is necessary to evaluate the population coverage, quality of services, appropriate documentation, and correct referral and follow up of high-risk people (3). This paper presents the findings of population screening coverage in the Kalutara district.

\section{Methodology}

The data relevant to coverage and quality of care were obtained by retrieving information from the WW Clinic registers maintained in the respective $\mathrm{MOH}$ areas.

Number of registrations, visual inspections and Pap smears performed each year from 1996 to 2000 were collected and these data were stratified to three different age groups ( $<39$ yrs, $40-59$ yrs, $>60$ yrs). The details of each WWClinic such as the year of commencement, availability of trained staff and facilities were recorded.

\section{Results}

Three of the $7 \mathrm{MOH}$ areas conducted more than 4 WWClinics per month. Only one $\mathrm{MOH}$ area had one outreach WWClinic. Two $\mathrm{MOH}$ areas conducted WWClinics separately as a single clinic, and the other five $\mathrm{MOH}$ areas conducted the clinics in combination with Maternal and Child Health / Family Planning clinics.

The statistics from the Deputy Provincial Director of Health Kalutara showed that there were 69225 females between 40-60 years of age, eligible for cervical screening in the district. Cervical screening is either visual inspection or visual inspection and Pap smear testing. Registrations and cervical screening carried out in W.W.Clinics in $\mathrm{MOH}$ areas by year is shown in figure 2 .

Figure 2 - Registrations and cervical screening carried out in the WWClinics

in Kalutara District by year

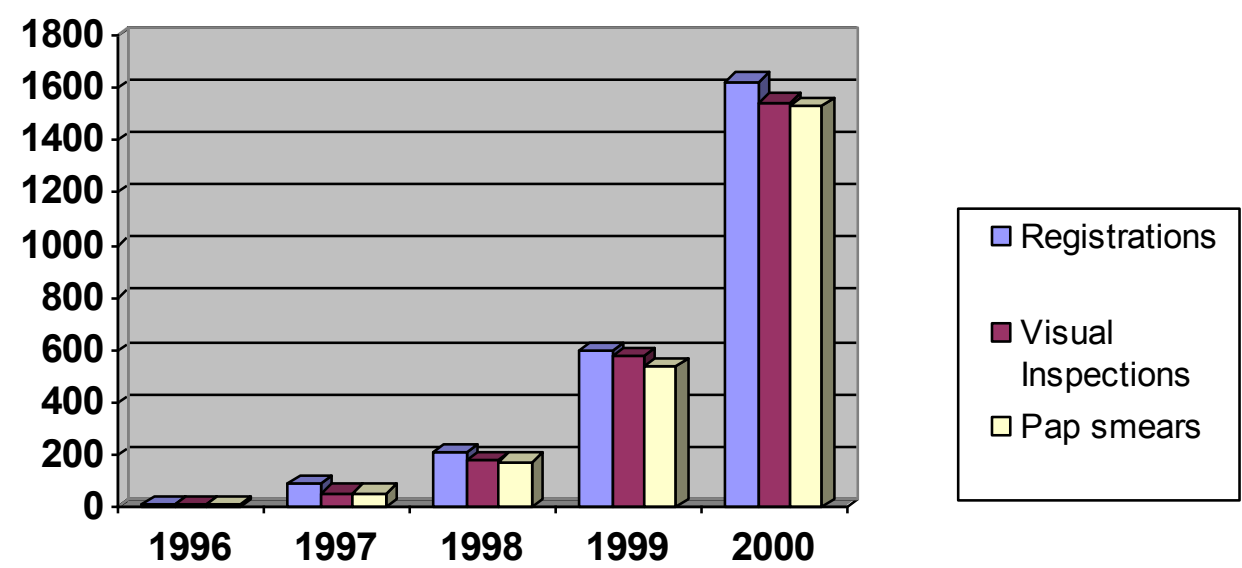


Although few cervical screening had been carried out initially it was observed to improve gradually with time and markedly after the year 1998 . The increasing trend of cervical screening observed in the district, from 1996 to 2000 is statistically significant (Visual Inspections$\mathrm{c}^{2}$ for trend $=3396, \mathrm{df}=4, \mathrm{p}=.000$ and for Pap Smear $\mathrm{c}^{2}$ for trend $=3408, \mathrm{df}=4, \mathrm{p}=.000)$.

The highest percentage of clients was registered in $\mathrm{Bu}-$ lathsinhala $\mathrm{MOH}$ area $(27.1 \%)$ and the lowest in Walallawita (4\%).

Out of the 2373 screened females in the district, Pap smears were performed among 2310 (91\%) clients and visual inspections had been carried out on the other 63 (9\%). The cervical screening started in 1996 in Agalawatta $\mathrm{MOH}$ area and it was restricted to visual inspections without Pap smear testing due to lack of equipment. For the period of study maximum number of Pap smears $(29.8 \%)$ was taken at Bulathsinghala and minimum at Bandaragama (3.9\%).
Percentage registrations of clients by age group in the WWClinics from 1996 to 2000 are illustrates in figure 3 .

The registration of target age group (40-60 yrs) had increased from $23 \%$ in 1996 to $68 \%$ in 2000 while registration of females under 39 years had declined to $25 \%$ from $69 \%$ for the same period as par with departmental guidelines.

\section{Evaluation of cervical screening coverage}

Cervical screening coverage for the period 19962000, in individual $\mathrm{MOH}$ areas and in Kalutara district is shown in table I.

Figure 3 - \% of clients registered in W.W.Clinics in Kalutara District by age and year

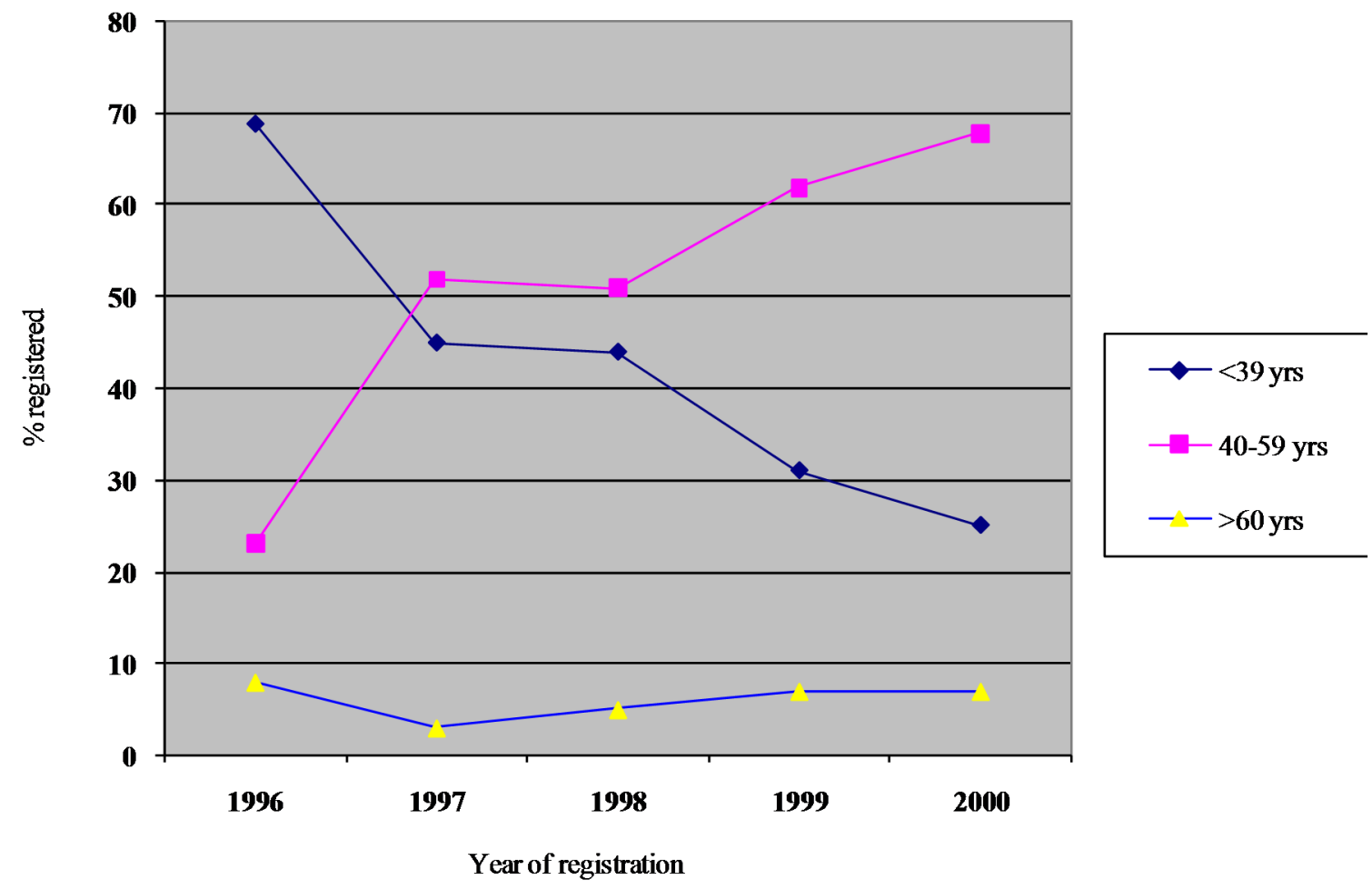




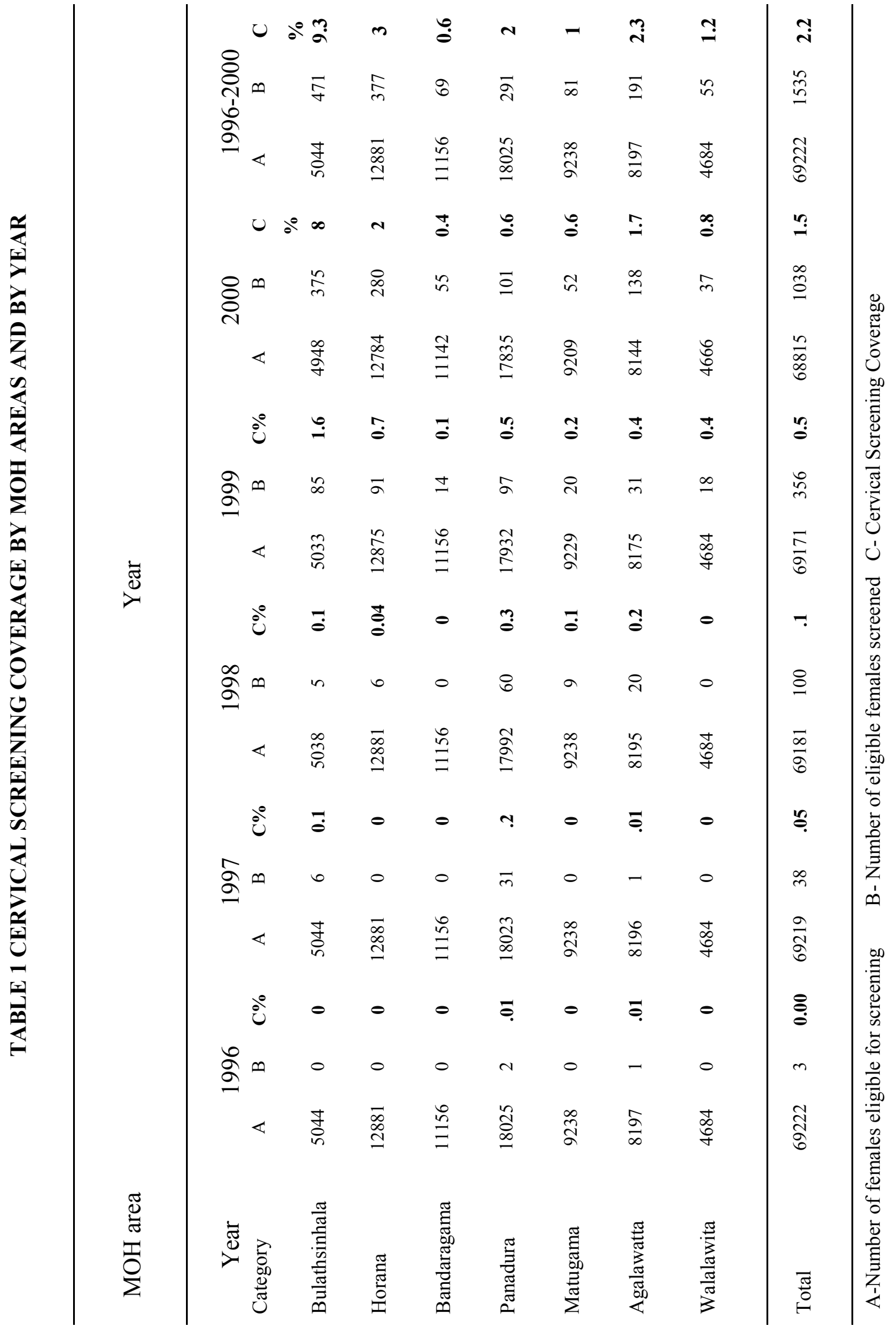


Cervical screening coverage in the Kalutara district is $1.5 \%$ in 2000 . Cumulative coverage for the five-year period (1996-2000) is $2.2 \%$, the highest cumulative coverage of $9.3 \%$ is observed in Bulathsinhala $\mathrm{MOH}$ area, where WWClinic conducted as a single clinic.

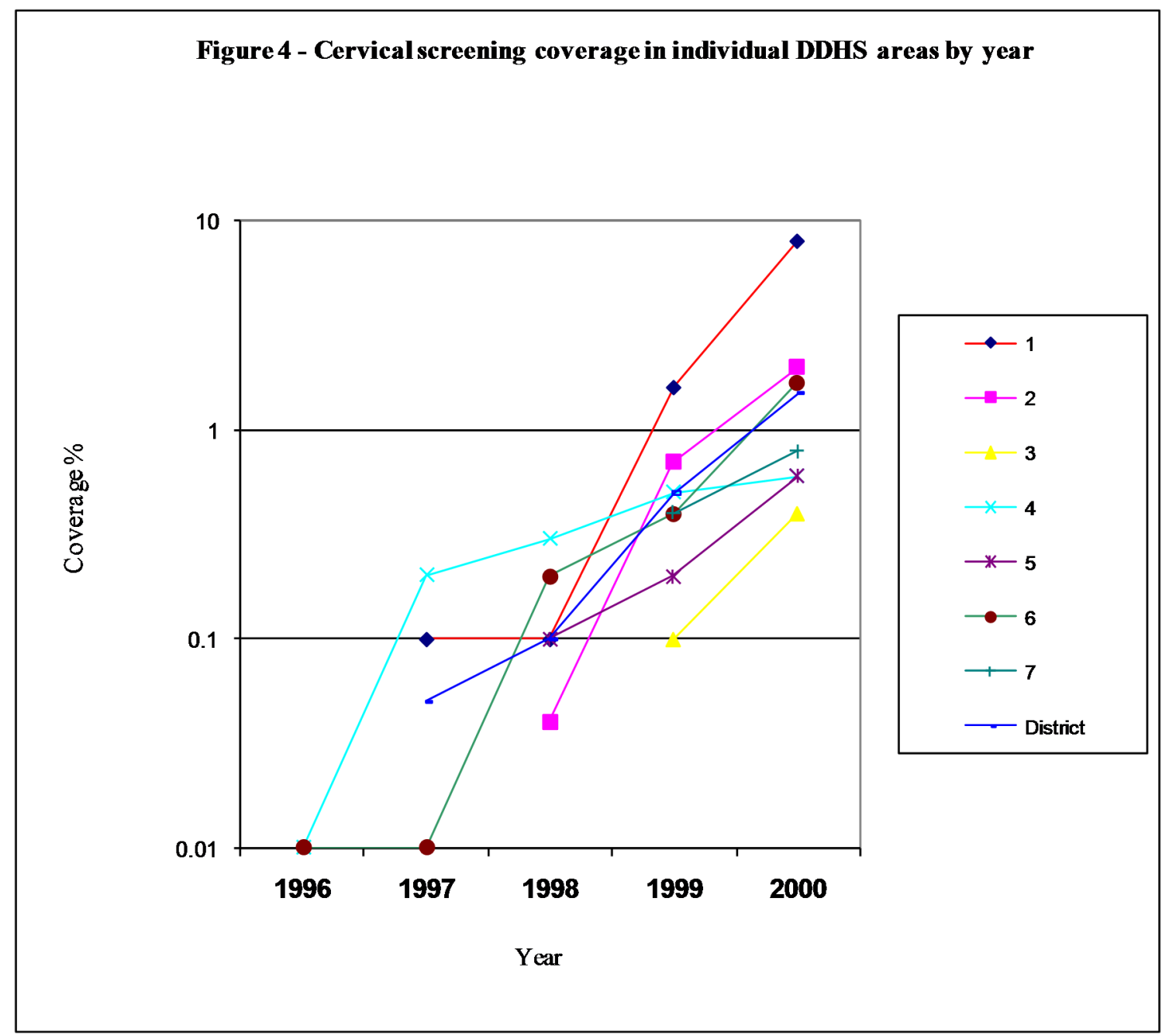

Although the screening coverage is low there is a gradual improvement over the years and this trend

Figure 5 - Distribution of trained is shown in figure 4.

MOOH and PHNSS in Kalutara

District 1996-2000

Availability of trained staff to perform cervical screening

Distribution of trained staff to perform cervical screening from the year 1996 to 2000 is shown in figure 5 .

$\mathrm{MOOH}$ were trained on cervical screening from the year 1996 onwards. Subsequently Public Health Nursing sisters too were trained to perform cervical screening and by 1998 all the $\mathrm{MOH}$ areas in the district had trained PHNSS (figure5).

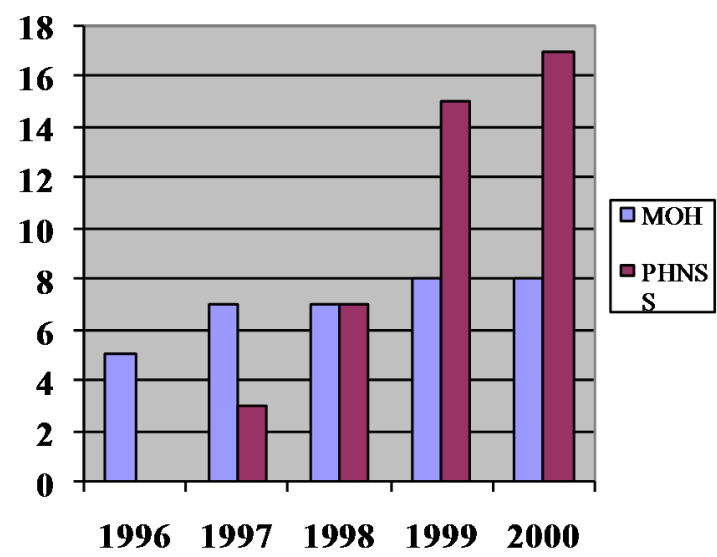




\section{Discussion}

The cervical screening coverage pattern resembles with that of other developing countries where only $5 \%$ of the target females had been screened in a five-year period (1). Hence, programme modification is needed to increase the cervical screening coverage.

Although the coverage was low, the present study observed an obvious rising trend of cervical screening from 1996, mostly marked after the year 1998 with the training of PHNSS in Kalutara District. Therefore PHNSS training could be considered as a useful strategy to increase cervical screening coverage.

Out of the total 2373 cervical screenings carried out during 1996 to 2000 in WWClinics, 172 (3\%) were identified as abnormal on visual inspection. Therefore visual inspection of cervix is a useful alternative strategy to Pap smear testing. This has been recommended by World Health Organization in resource poor setting (5). Accordingly, one $\mathrm{MOH}$ area only visual inspections had been carried out for some time till the equipment was available.

In $1996,23 \%$ of the clients were between $40-60$ years of age which was the target age of the screening programme. In 2000, it had risen to $68 \%$ indicating that the programme was progressing to achieve the objectives of the National Cervical Screening Programme laid down by the Ministry of Health. Since the development of cervical cancer directly related to the sexual activity, it is recommended to lower the inception of screening to 35 years.

Low cervical screening coverage could be rectified by increasing frequency and number of clinics especially in the periphery. Highest cervical screening coverage was shown in an area where the WWClinic was conducted as a single clinic. Hence, it shows the necessity of conducting W.W.Clinics on separate days without combining with $\mathrm{MCH} / \mathrm{FP}$ Clinics.

\section{Acknowledgement}

The authors would like to extend their sincere gratitude to National Science Foundation for providing financial assistance to carry out the study.

\section{References}

1. Cancer Control Programme. National Cancer Institute Sri Lanka. 1990.

2. Cancer Incidence. Cancer Control Programme. National Cancer Institute Sri Lanka.1995

3. Park 1997 Park, K. Epidemiology of Chronic Non-Communicable Diseases and conditions. Text book of Preventive and Social Medicine. $199715^{\text {th }}$ edn. M/S Banarsides Bhanot. Jabalpur (India) 286.

4. Laara,E., Day,N.E and Hakama, M., Trends in mortality from cervical cancer in The Nordic countries:Association with organized screening programme. The Lancet. 1987; ( 1)1247-1249.

5. Programme for Appropriate Technology in Health. Preventing Cervical Cancer in Lowresource settings. Out Look .1998; ;6 (1) 1-4. 